\title{
Design, Synthesis and Docking Studies of New 4-hydroxyquinoline-3-carbohydrazide Derivatives as Anti-HIV-1 Agents
}

Authors

Affiliations

\author{
Z. Hajimahdi ${ }^{1}$, R. Zabihollahi ${ }^{2}$, M. R. Aghasadeghi ${ }^{2}$, A. Zarghi ${ }^{1}$
}

${ }^{1}$ Department of Medicinal Chemistry, School of Pharmacy, Shahid Beheshti University of Medical Sciences, Tehran, Iran ${ }^{2}$ Hepatitis and AIDS department, Pasteur institute of Iran, Tehran, Iran

\author{
Key words \\ - synthesis \\ - docking studies \\ - 4-Hydroxyquinoline-3- \\ carbohydrazide \\ - anti-HIV-1 activity
}

received 18.11.2012

accepted 31.01.2013

\section{Bibliography}

DOI http://dx.doi.org/ 10.1055/s-0033-1334964

Published online:

March 13, 2013

Drug Res 2013;

63: 192-197

(c) Georg Thieme Verlag KG

Stuttgart · New York

ISSN 2194-9379

\section{Correspondence}

\section{A. Zarghi}

Department of Pharmaceutical Chemistry

School of Pharmacy

Shahid Beheshti University of

Medical Sciences

P.O.Box: 14155-6153

Tehran

Iran

Tel.: + 98/21/8820 0096

Fax: + 98/21/8866 5341

zarghi@sbmu.ac.ir

\section{Abstract}

$\nabla$

A new class of 4-hydroxyquinoline-3-carbohydrazide derivatives was prepared and evaluated for its anti-HIV activity. The primary bioassay results indicated that most of tested compounds possess moderate inhibitory properties against HIV-1 virus (NL4-3) in Hela cells cultures. Our results also indicated that compounds $\mathbf{6} \mathbf{d}$ and $\mathbf{7 e}$ were the most potent anti-HIV agents among the synthesized compounds with inhibition rate of 32 and $28 \%$ at concentration of $100 \mu \mathrm{M}$, respec-

\section{Introduction}

$\nabla$

The causative agent of acquired immune deficiency syndrome (AIDS) is the human immunodeficiency virus type 1 (HIV-1). During the past 3 decades, the combination of antiretroviral drugs in HAART (highly active antiretroviral therapy) regimens has transformed the management of HIV infection from a fatal disease to a manageable chronic condition $[1,2]$. However, resistance to marketed antiHIV drugs is increasing at an alarming rate. Thus, there is a need to develop new agents which work by different mechanisms. In this regard, there has been considerable interest in HIV integrase (IN) function as an attractive alternative target that may lead to the next generation of anti-HIV drug [3,4]. HIV-1 integrase is one of the 3 most important enzymes required for viral replication. It catalyzes the insertion and the integration of the proviral DNA into the genome of the host cell in 2 steps: 3 '-processing (3'-P), the endonucleolytic sequencespecific hydrolysis of 3'-ends of the viral cDNA, and strand transfer (ST), the ligation of the viral 3'-OH cDNA ends to the phosphate backbone of the host DNA acceptor. This enzymatic process is dependent on an active site containing dual $\mathrm{Mg}^{2+}$ metal ions held in place with a highly conserved triad of carboxylate amino acid residues (asp64/asp116/ glu152) commonly referred to as a $\mathrm{DD}(35) \mathrm{E}$ triad tively. A docking study using the later crystallographic data available for PFV integrase including its complexes with $\mathrm{Mg}^{2+}$ and raltegravir, showed that the designed compounds bind into the active site of integrase such that carboxylic and hydroxyl groups of 4-hydroxyquinoline-3-carbohydrazide chelate the $\mathrm{Mg}^{2+}$ ion. Interestingly, all of the synthesized compounds were found to present no significant cytotoxicity at concentration of $100 \mu \mathrm{M}$. Therefore, these compounds can provide a very good basis for the development of new anti-HIV-1 agents.
[5-7]. Thus the chelation of the critical $\mathrm{Mg}^{2+}$ cofactors can cause the functional impairment of IN. In fact, all of the small molecule HIV-1 IN inhibitors commonly contain a structural motif that coordinates the 2 divalent magnesium ions in the enzyme's active site [8]. Typically, the most developed and promising $\beta$-diketoacid, naphthyridine carboxamide, pyrimidinone and quinolone carboxylic acid classes of IN inhibitors belong to this category [9]. Among these chelation inhibitors, raltegravir has been approved by the FDA, GS-9137 (Elvitegravir) and S/GSK-1349572 ( $\bullet$ Fig. 1) have reached clinical development [10,11]. Moreover, emergence of resistance against raltegravir due to viral mutations demands exploration of novel scaffolds for the treatment of HIV infection [12]. In this research, aimed at the discovery of new compounds as anti-HIV-1 agents, we selected the HIV-1 integrase inhibitors as a lead to design new analogues. In order to target the catalytic site of HIV-1 integrase, we designed new IN inhibitors by merging the pharmacophores of the salicylhydrazide and 4-quinolone-3-carboxylic acid to generate novel chemical scaffolds. The salicylhydrazide and polyhydroxyl aromatics (as exemplified by the structures ( 1 \& 2) in $\odot$ Fig. 1) were previously reported as potent IN inhibitors, but the inherently high cytotoxicity in these compounds limited their therapeutic application as 
<smiles>Cc1nnc(C(=O)NC(C)(C)c2nc(C(=O)NCc3ccc(F)cc3)c(O)c(=O)n2C)o1</smiles>

Raltegravir<smiles>Cc1c(Cl)cccc1Cc1cc2c(=O)c(C(=O)O)cn(C[C@H](O)C(C)C)c2cc1O</smiles>

Elvitegravir<smiles>C[C@@H]1CCO[C@]2(C)Cn3cc(C(=O)NCc4ccc(F)cc4F)c(=O)c(O)c3C(=O)N12</smiles>

S/GSK-1349572<smiles>O=C(NNC(=O)c1ccc2ccccc2c1O)c1ccccc1O</smiles>

1<smiles>Cc1ccc(-n2c(SCC(=O)NNC(=O)c3ccccc3O)nnc2-c2ccccc2O)cc1</smiles>

2<smiles>[Y]C(=O)NNC(=O)c1cnc2ccccc2c1O</smiles>

Designed compounds (6a-e, 7a-e)

Fig. 1 HIV-1 integrase inhibitors (Raltegravir, Elvitegravir, S/GSK-1349572), lead compounds (1, 2) and designed scaffold.

antiretroviral agents [13-16]. On the other hand, 4-quinolone-3carboxylic acid derivatives are attractive in anti-HIV-1 drug discovery due to their extremely versatile nature, well-known biochemical properties and having no significant cytotoxic effects $[17,18]$. Based on these facts supported by literature and as a part of our research program aimed at discovering new anti-HIV-1 agents, we designed a novel noncytotoxic class of 4-hydroxyquinoline-3-carbohydrazide derivatives with various substituted aromatic moiety by integration of fragments of salicylhydrazide and 4-quinolone-3-carboxylic acid. Actually, according to HIV-1 integrase inhibitors structure activity relationship, the adjacent carboxylic and hydroxyl groups on 4-hydroxyquinoline-3-carbohydrazide could serve as the metal binding pharmacophore. Furthermore, the aryl group might serve as the pharmacophore to interact with the hydrophobic binding surface of the IN. We also performed docking studies to predict the interaction of new synthesized compounds into the active site of integrase and their probable mechanism of action.

\section{Material and Methods \\ $\nabla$}

\section{Materials}

All chemicals, reagents and solvents used in this study were purchased from Merck AG and Aldrich Chemical. Melting points were determined with a Thomas-Hoover capillary apparatus. A Synthos 3000 microwave oven (Anton Paar, Austria) was used for synthesis of compounds. Infrared spectra were acquired using a Perkin Elmer Model 1420 spectrometer. A Bruker FT-500MHz instrument (Brucker Biosciences, USA) was used to acquire ${ }^{1}$ HNMR spectra with
TMS as internal standard. Chloroform-D and DMSO- $\mathrm{D}_{6}$ were used as solvents. The mass spectral measurements were performed on an 6410 Agilent LCMS triple quadrupole mass spectrometer (LCMS) with an electrospray ionization (ESI) interface. Microanalyses, determined for $\mathrm{C}$ and $\mathrm{H}$, were within $\pm 0.4 \%$ of theoretical values.

\section{Preparation of diethyl 2-((phenylamino)methylene) malonate (3)}

A mixture of aniline $(0.9 \mathrm{ml}, 10 \mathrm{mmol})$ and diethyl ethoxymethylenemalonate (EMME) $(2 \mathrm{ml}, 10 \mathrm{mmol})$ was heated at $120^{\circ} \mathrm{C}$ for $1 \mathrm{~h}$. The reaction mixture was cooled to room temperature. The solid thus formed was taken in n-hexane and stirred for $15 \mathrm{~min}$ and filtered to afford compound 3. Yield, $62 \%$; white powder; $\mathrm{mp} 44-45^{\circ} \mathrm{C}$ [19]; IR (KBr disk): $v$ (cm-1) 1400-1600 (aromatic), 1660, 1690 (C=O), 3300 (N-H); LC-MS (ESI) m/z: $286.12(\mathrm{M}+23,100)$.

Preparation of ethyl 4-hydroxyquinoline-3-carboxylate (4) A diphenyl ether solution of 3 containing catalytic 2-chlorobenzoic acid was heated by microwave irradiation $\left(250^{\circ} \mathrm{C}, 180 \mathrm{~W}\right)$ for $2 \mathrm{~h}$. The reaction mixture was cooled to room temperature and added n-hexane $(50 \mathrm{ml})$ and stirred for $15 \mathrm{~min}$, the precipitated solid was filtered and dried to get compound $\mathbf{4}$. The final product was crystallized in ethanol. Yield, 75\%; cream powder; $\mathrm{mp} 261-262^{\circ} \mathrm{C}$ [19]; IR (KBr disk): $v\left(\mathrm{~cm}^{-1}\right)$ 1400-1600 (aromatic), 1695 (C=O), 3150 (N-H); LC-MS (ESI) m/z: $218.08(\mathrm{M}+1,100)$.

Preparation of 4-hydroxyquinoline-3-carbohydrazide (5) A mixture of $4(2 \mathrm{~g}, 4.5 \mathrm{mmol})$ and hydrazine hydrate $(6 \mathrm{ml}, 45 \mathrm{mmol})$ in $\mathrm{DMF}(5 \mathrm{ml})$ was stirred at room temperature for $12 \mathrm{~h}$. Completion 
of the reaction was monitored by TLC. After reaction completion, water $(50 \mathrm{ml})$ was added and the solid product obtained was filtered, washed with water to give $\mathbf{5}$. The final product was crystallized in ethanol. Yield, 55\%; white powder; $\mathrm{mp} 290-292^{\circ} \mathrm{C}$ (decomposed); IR (KBr disk): $\mathrm{v}\left(\mathrm{cm}^{-1}\right)$ 1400-1600 (aromatic), $1730(\mathrm{C}=0), 2500-3400$ (O-H), $3310(\mathrm{~N}-\mathrm{H}), 3370\left(\mathrm{NH}_{2}\right)$; LC-MS (ESI) m/z: 205.07 (M+1, 100).

General procedure for preparation of 4-hydroxy-N'-(4substituted-benzoyl) quinoline -3-carbohydrazide (6a-e) A mixture of $\mathbf{5}(0.3 \mathrm{~g}, 1.5 \mathrm{mmol})$, 4-substituted benzoyl chlorides $(0.2 \mathrm{ml}, 1.5 \mathrm{mmol})$ and a catalytic amount of $\mathrm{Na}_{2} \mathrm{CO}_{3}(0.05 \mathrm{~g}$, $0.5 \mathrm{mmol})$ was dissolved in dry DMF $(10 \mathrm{ml})$ and stirred at room temperature for $16 \mathrm{~h}$. The reaction mixture was slowly poured over crushed ice and kept for $2 \mathrm{~h}$. The solid thus separated out was filtered, washed with water and recrystallized from ethanol to give compounds (6a-e) (Yield, 30-40\%).

N'-Benzoyl-4-hydroxyquinoline-3-carbohydrazide (6a) Yield, 40\%; white powder; mp $288-290^{\circ} \mathrm{C}$ (decomposed); IR ( $\mathrm{KBr}$ disk): $\mathrm{v}$ ( $\left.\mathrm{cm}^{-1}\right)$ 1400-1600 (aromatic), 1630-1680 (C=0), 2800-3200 (O-H), $3210(\mathrm{~N}-\mathrm{H}) ;{ }^{1} \mathrm{HNMR}$ (DMSO-d6, 500MHz): $\delta$ 7.46-7.51 (m, 3H, benzoyl $\mathrm{H}_{3}-\mathrm{H}_{5}$ ), 7.54-7.57 (t, $1 \mathrm{H}$, quinoline $\mathrm{H}_{6}$, $J=7.26 \mathrm{~Hz}), 7.71\left(\mathrm{~d}, 1 \mathrm{H}\right.$, quinoline $\left.\mathrm{H}_{8}, J=8.07 \mathrm{~Hz}\right), 7.76-7.79(\mathrm{~m}, 1 \mathrm{H}$, quinoline $\mathrm{H}_{7}$ ), 7.88 (d, $2 \mathrm{H}$, benzoyl $\left.\mathrm{H}_{2} \& \mathrm{H}_{6}, J=7.16 \mathrm{~Hz}\right), 8.27$ (d, $1 \mathrm{H}$, quinoline $\left.\mathrm{H}_{5}, J=8.09 \mathrm{~Hz}\right), 8.77\left(\mathrm{~s}, 1 \mathrm{H}\right.$, quinoline $\left.\mathrm{H}_{2}\right), 10.75(\mathrm{~d}, 1 \mathrm{H}, \mathrm{N}-\mathrm{H}$, $J=2.7 \mathrm{~Hz}$ ), 11.70 (d, 1H, N-H, J=2.7Hz), 12.83 (br s, $1 \mathrm{H}$, enolic $\mathrm{OH}$ ); LC-MS (ESI) m/z: $307.80(\mathrm{M}+1,100)$; Anal. Calcd. for $\mathrm{C}_{17} \mathrm{H}_{13} \mathrm{~N}_{3} \mathrm{O}_{3}$ : C, $66.44 ; \mathrm{H}, 4.26$; N, 13.67. Found: C, 66.64; H, 4.49; N, 13.60 .

\section{N'-(4-Fluorobenzoyl)-4-hydroxyquinoline-3-}

carbohydrazide (6b)

Yield, 30\%; white powder; $\mathrm{mp} 280^{\circ} \mathrm{C}$ (decomposed); IR ( $\mathrm{KBr}$ disk): $\mathrm{v}\left(\mathrm{cm}^{-1}\right)$ 1400-1600 (aromatic), 1640-1675 (C=0), 2900-3400 (O-H), 3400 (N-H); ${ }^{1} \mathrm{HNMR}$ (DMSO-d6, 500MHz): $\delta 7.30-7.33(\mathrm{t}$, $2 \mathrm{H}$, 4-F-benzoyl $\mathrm{H}_{3} \& \mathrm{H}_{5}, J=8.84 \mathrm{~Hz}$ ), 7.47-7.50 (t, quinoline $\mathrm{H}_{6}$, $J=7.88 \mathrm{~Hz}$ ), $7.71\left(\mathrm{~d}, 1 \mathrm{H}\right.$, quinoline $\left.\mathrm{H}_{8}, J=8.14 \mathrm{~Hz}\right), 7.76-7.79(\mathrm{t}, 1 \mathrm{H}$, quinoline $\left.\mathrm{H}_{7}, J=7.04 \mathrm{~Hz}\right), 7.94-7.97\left(\mathrm{~m}, 2 \mathrm{H}, 4\right.$-F-benzoyl $\left.\mathrm{H}_{2} \& \mathrm{H}_{6}\right)$, $8.28\left(\mathrm{~d}, 1 \mathrm{H}\right.$, quinoline $\left.\mathrm{H}_{5}, J=7.47 \mathrm{~Hz}\right), 8.76\left(\mathrm{~s}, 1 \mathrm{H}\right.$, quinoline $\left.\mathrm{H}_{2}\right)$, 10.80 (s, 1H, N-H), 11.72 (s, 1H, N-H), 12.83 (br s, 1H, enolic OH); LC-MS (ESI) m/z: $325.81(\mathrm{M}+1,100)$; Anal. Calcd. for $\mathrm{C}_{17} \mathrm{H}_{12} \mathrm{FN}_{3} \mathrm{O}_{3}$ : C, 62.77; H, 3.72; N, 12.92. Found: C, 62.54; H, 3.56; N, 13.01.

N'-(4-Chlorobenzoyl)-4-hydroxyquinoline-3-

carbohydrazide (6c)

Yield, 40\%; white powder; $\mathrm{mp} 290^{\circ} \mathrm{C}$ (decomposed); IR (KBr disk): $\mathrm{v}$ $\left(\mathrm{cm}^{-1}\right.$ ) 1400-1600 (aromatic), 1640-1670 (C=0), 2800-3300 (O-H); ${ }^{1} \mathrm{HNMR}$ (DMSO-d6, 500MHz): $\delta$ 7.47-7.50 (t, quinoline $\mathrm{H}_{6}$, $J=7.76 \mathrm{~Hz}$ ), 7.56 (d, 2H, 4-Cl-benzoyl $\mathrm{H}_{3} \& \mathrm{H}_{5}, J=8.52 \mathrm{~Hz}$ ), 7.71 (d, $1 \mathrm{H}$, quinoline $\left.\mathrm{H}_{8}, J=8.14 \mathrm{~Hz}\right), 7.75-7.79\left(\mathrm{t}, 1 \mathrm{H}\right.$, quinoline $\left.\mathrm{H}_{7}, J=8.24 \mathrm{~Hz}\right)$, 7.90 (d, 2H, 4-Cl-benzoyl $\mathrm{H}_{2} \& \mathrm{H}_{6}, J=8.60 \mathrm{~Hz}$ ), 8.27 (d, $1 \mathrm{H}$, quinoline $\left.\mathrm{H}_{5}, J=7.84 \mathrm{~Hz}\right), 8.76\left(\mathrm{~d}, 1 \mathrm{H}\right.$, quinoline $\left.\mathrm{H}_{2}, J=6.7 \mathrm{~Hz}\right), 10.87(\mathrm{~d}, 1 \mathrm{H}, \mathrm{N}-\mathrm{H}$, $J=2.7 \mathrm{~Hz}$ ), 11.75 (d, 1H, N-H), 12.83 (d, $1 \mathrm{H}$, enolic $\mathrm{OH}, J=6.45 \mathrm{~Hz}$ ); LC-MS (ESI) m/z: $342.04(\mathrm{M}+1,100)$; Anal. Calcd. for $\mathrm{C}_{17} \mathrm{H}_{12} \mathrm{ClN}_{3} \mathrm{O}_{3}$ : C, 59.75; H, 3.54; N, 12.30. Found: C, 59.99; H, 3.36; N, 12.57 .

\section{4-Hydroxy-N'-(4-methylbenzoyl)quinoline-3-}

carbohydrazide (6d)

Yield, 30\%; white powder; mp 299-301 ${ }^{\circ} \mathrm{C}$; IR (KBr disk): v $\left(\mathrm{cm}^{-1}\right)$ 1400-1600 (aromatic), 1640-1690 (C= O), 2900-3300 (O-H); ${ }^{1} \mathrm{HNMR}$ (DMSO-d6, $500 \mathrm{MHz}$ ): $\delta 2.34$ (s, 3H, $\mathrm{CH}_{3}$ ), 7.28 (d, $2 \mathrm{H}$, p-Toluoyl $\mathrm{H}_{3} \& \mathrm{H}_{5}, J=8.01 \mathrm{~Hz}$ ), 7.47-7.50 (t, quinoline $\mathrm{H}_{6}$, $J=7.17 \mathrm{~Hz}), 7.71\left(\mathrm{~d}, 1 \mathrm{H}\right.$, quinoline $\left.\mathrm{H}_{8}, J=8.07 \mathrm{~Hz}\right), 7.75-7.80(\mathrm{~m}$,
$3 \mathrm{H}$, quinoline $\mathrm{H}_{7}$ and 4 p-Toluoyl $\mathrm{H}_{2} \& \mathrm{H}_{6}$ ), 8.27 (d, $1 \mathrm{H}$, quinoline $\mathrm{H}_{5}, J=8.06 \mathrm{~Hz}$ ), $8.76\left(\mathrm{~s}, 1 \mathrm{H}\right.$, quinoline $\left.\mathrm{H}_{2}\right), 10.66(\mathrm{~s}, 1 \mathrm{H}, \mathrm{N}-\mathrm{H})$, 11.68 (s, 1H, N-H), 12.82 (s, 1H, enolic OH); LC-MS (ESI) m/z: $321.92(\mathrm{M}+1,100)$; Anal. Calcd. for $\mathrm{C}_{18} \mathrm{H}_{15} \mathrm{~N}_{3} \mathrm{O}_{3}$ : C, 67.28; $\mathrm{H}$, $4.71 ; \mathrm{N}, 13.08$. Found: C, 67.44; H, 4.87; N, 13.17.

\section{4-Hydroxy-N'-(4-methoxybenzoyl)quinoline-3-}

carbohydrazide (6e)

Yield, 30\%; white powder; mp 260-262 ${ }^{\circ} \mathrm{C}$; IR ( $\mathrm{KBr}$ disk): $\mathrm{v}$ $\left(\mathrm{cm}^{-1}\right.$ ) 1400-1600 (aromatic), 1630-1685 (C= O), 2800-3200 (O-H), 3370 \& 3200 (N-H); ${ }^{1} \mathrm{HNMR}$ (DMSO-d6, $500 \mathrm{MHz}$ ): $\delta 3.79$ (s, $3 \mathrm{H}, \mathrm{OCH}_{3}$ ), 7.00 (d, $2 \mathrm{H}, 4-\mathrm{OCH}_{3}$-benzoyl $\mathrm{H}_{3} \& \mathrm{H}_{5}, J=8.76 \mathrm{~Hz}$ ), 7.47-7.50 (t, quinoline $\left.\mathrm{H}_{6}, J=7.79 \mathrm{~Hz}\right), 7.71\left(\mathrm{~d}, 1 \mathrm{H}\right.$, quinoline $\mathrm{H}_{8}$, $J=8.18 \mathrm{~Hz}$ ), 7.75-7.79 (t, $1 \mathrm{H}$, quinoline $\left.\mathrm{H}_{7}, J=8.22 \mathrm{~Hz}\right), 7.87$ (d, $2 \mathrm{H}, 4-\mathrm{OCH}_{3}$-benzoyl $\left.\mathrm{H}_{2} \& \mathrm{H}_{6}, J=8.73 \mathrm{~Hz}\right), 8.27(\mathrm{~d}, 1 \mathrm{H}$, quinoline $\left.\mathrm{H}_{5}, J=7.90 \mathrm{~Hz}\right), 8.75$ (d, $1 \mathrm{H}$, quinoline $\left.\mathrm{H}_{2}, J=6.61 \mathrm{~Hz}\right), 10.59(\mathrm{~s}, 1 \mathrm{H}$, $\mathrm{N}-\mathrm{H}), 11.65(\mathrm{~s}, 1 \mathrm{H}, \mathrm{N}-\mathrm{H}), 12.81$ (d, $1 \mathrm{H}$, enolic $\mathrm{OH}, J=6.3 \mathrm{~Hz}$ ); LC-MS (ESI) m/z: $337.82(\mathrm{M}+1,100)$; Anal. Calcd. for $\mathrm{C}_{18} \mathrm{H}_{15} \mathrm{~N}_{3} \mathrm{O}_{4}$ : C, 64.09; H, 4.48; N, 12.46. Found: C, 64.24; H, 4.31; N, 12.56.

General procedure for preparation of N-(4-substitutedphenyl)-2-(4-hydroxy quinoline-3-carbonyl)

hydrazinecarboxamide (7a-e)

a mixture of $\mathbf{5}(0.3 \mathrm{~g}, 1.5 \mathrm{mmol})$ and 4 -substituted phenyl isocyanates $(0.2 \mathrm{ml}, 1.5 \mathrm{mmol})$ was dissolved in acetonitrile $(10 \mathrm{ml})$ and stirred at room temperature for $16 \mathrm{~h}$. The reaction mixture was slowly poured over crushed ice and kept for $2 \mathrm{~h}$. The solid thus separated out was filtered, washed with water and recrystallized from ethanol to give compounds (7a-e) (yield, 30-40\%)

\section{2-(4-Hydroxyquinoline-3-carbonyl)-N-}

phenylhydrazinecarboxamide (7a)

Yield, $35 \%$; white powder; $\mathrm{mp} 250-252^{\circ} \mathrm{C}$; IR (KBr disk): $\mathrm{v}\left(\mathrm{cm}^{-1}\right)$ 1400-1600 (aromatic), 1645-1670 (C=O), 2800-3500 (O-H), 3290,3340 \& 3470 (N-H); ${ }^{1} \mathrm{HNMR}$ (DMSO-d6, 500MHz): $\delta 6.89-$ $6.92\left(\mathrm{t}, 1 \mathrm{H}, \mathrm{N}\right.$-phenyl $\left.\mathrm{H}_{4}, J=7.36 \mathrm{~Hz}\right), 7.20-7.23\left(\mathrm{t}, 2 \mathrm{H}, \mathrm{N}\right.$-phenyl $\mathrm{H}_{3}$ \& $\left.\mathrm{H}_{5}, J=7.59 \mathrm{~Hz}\right), 7.41\left(\mathrm{~d}, 2 \mathrm{H}, \mathrm{N}-\right.$ phenyl $\left.\mathrm{H}_{2} \& \mathrm{H}_{6}, J=7.71 \mathrm{~Hz}\right), 7.46-$ 7.49 (t, quinoline $\left.\mathrm{H}_{6}, J=7.14 \mathrm{~Hz}\right), 7.69\left(\mathrm{~d}, 1 \mathrm{H}\right.$, quinoline $\mathrm{H}_{8}$, $J=8.06 \mathrm{~Hz}), 7.74-7.77\left(\mathrm{t}, 1 \mathrm{H}\right.$, quinoline $\left.\mathrm{H}_{7}, J=6.98 \mathrm{~Hz}\right), 8.26(\mathrm{~d}, 1 \mathrm{H}$, quinoline $\left.\mathrm{H}_{5}, J=7.02 \mathrm{~Hz}\right), 8.40(\mathrm{~s}, 1 \mathrm{H}, \mathrm{N}-\mathrm{H}), 8.73(\mathrm{~s}, 1 \mathrm{H}$, quinoline $\mathrm{H}_{2}$ ), 8.83 (s, $1 \mathrm{H}, \mathrm{N}-\mathrm{H}$ ), 11.39 (s, $1 \mathrm{H}, \mathrm{N}-\mathrm{H}$ ), 12.83 (br s, $1 \mathrm{H}$, enolic OH); LC-MS (ESI) m/z: 322.91 (M+1,100); Anal. Calcd. for $\mathrm{C}_{17} \mathrm{H}_{14} \mathrm{~N}_{4} \mathrm{O}_{3}$ : C, 63.35; H, 4.38; N, 17.38. Found: C, 63.44; H, 4.29; N, 17.52 .

\section{N-(4-Fluorophenyl)-2-(4-hydroxyquinoline-3-carbonyl)}

hydrazinecarboxamide (7b)

Yield, 35\%; white powder; $\mathrm{mp} 309-310^{\circ} \mathrm{C}$; IR ( $\mathrm{KBr}$ disk): $\mathrm{v}$ $\left(\mathrm{cm}^{-1}\right)$ 1400-1600 (aromatic), 1620-1680 (C=0), 2900-3500 $(\mathrm{O}-\mathrm{H}), 3290 \& 3450(\mathrm{~N}-\mathrm{H}) ;{ }^{1} \mathrm{HNMR}$ (DMSO-d6, 500 MHz): $\delta$ 7.03-7.07 (t, 2H, N-4-fluorophenyl $\mathrm{H}_{3} \& \mathrm{H}_{5}, J=8.86 \mathrm{~Hz}$ ), 7.42$7.44\left(\mathrm{~m}, 2 \mathrm{H}, \mathrm{N}-4\right.$-fluorophenyl $\mathrm{H}_{2} \& \mathrm{H}_{6}$ ), 7.46-7.49 (t, quinoline $\left.\mathrm{H}_{6}, J=7.60 \mathrm{~Hz}\right), 7.69\left(\mathrm{~d}, 1 \mathrm{H}\right.$, quinoline $\left.\mathrm{H}_{8}, J=8.22 \mathrm{~Hz}\right), 7.74-7.77$ (t, $1 \mathrm{H}$, quinoline $\left.\mathrm{H}_{7}, J=7.59 \mathrm{~Hz}\right), 8.26\left(\mathrm{~d}, 1 \mathrm{H}\right.$, quinoline $\mathrm{H}_{5}$, $J=8.00 \mathrm{~Hz}), 8.39(\mathrm{~s}, 1 \mathrm{H}, \mathrm{N}-\mathrm{H}), 8.73\left(\mathrm{~s}, 1 \mathrm{H}\right.$, quinoline $\left.\mathrm{H}_{2}\right), 8.87$ (s, 1H, N-H), 11.35 (s, 1H, N-H), 12.78 (br s, 1H, enolic OH); LC-MS (ESI) $\mathrm{m} / \mathrm{z}$ : $340.92(\mathrm{M}+1,100)$; Anal. Calcd. for $\mathrm{C}_{17} \mathrm{H}_{13} \mathrm{FN}_{4} \mathrm{O}_{3}: \mathrm{C}$, $60.00 ; \mathrm{H}, 3.85$; N, 16.46. Found: C, 60.14; H, 3.99; N, 16.67.

\section{N-(4-Chlorophenyl)-2-(4-hydroxyquinoline-3-carbonyl)} hydrazine carboxamide (7c)

Yield, $40 \%$; white powder; $\mathrm{mp} 279-280^{\circ} \mathrm{C}$; IR ( $\mathrm{KBr}$ disk): v $\left(\mathrm{cm}^{-1}\right)$ 1400-1600 (aromatic), 1650-1690 (C=0), 2900-3500 
(O-H), $3210 \& 3380(\mathrm{~N}-\mathrm{H}) ;{ }^{1} \mathrm{HNMR}$ (DMSO-d6, $\left.500 \mathrm{MHz}\right): \delta 7.25$ (d, 2H, N-4-chlorophenyl $\mathrm{H}_{3} \& \mathrm{H}_{5}, \mathrm{~J}=8.85 \mathrm{~Hz}$ ), 7.43-7.47 (m, 3H, quinoline $\mathrm{H}_{6} \& \mathrm{~N}-4$-chlorophenyl $\mathrm{H}_{2} \& \mathrm{H}_{6}$ ), 7.68 (d, $1 \mathrm{H}$, quinoline $\mathrm{H}_{8}, J=8.06 \mathrm{~Hz}$ ), 7.71-7.74 (t, $1 \mathrm{H}$, quinoline $\left.\mathrm{H}_{7}, J=7.14 \mathrm{~Hz}\right), 8.25$ (d, $1 \mathrm{H}$, quinoline $\mathrm{H}_{5}, J=7.56 \mathrm{~Hz}$ ), 8.43 (br s, $1 \mathrm{H}, \mathrm{N}-\mathrm{H}$ ), 8.73 (s, $1 \mathrm{H}$, quinoline $\left.\mathrm{H}_{2}\right), 8.98(\mathrm{~s}, 1 \mathrm{H}, \mathrm{N}-\mathrm{H}), 11.46(\mathrm{~s}, 1 \mathrm{H}, \mathrm{N}-\mathrm{H})$; LC-MS (ESI) $\mathrm{m} / \mathrm{z}$ : $356.85(\mathrm{M}+1,100)$; Anal. Calcd. for $\mathrm{C}_{17} \mathrm{H}_{13} \mathrm{ClN}_{4} \mathrm{O}_{3}$ : C, 57.23; H, 3.67; N, 15.70. Found: C, 57.46; H, 3.88; N, 15.79 .

\section{2-(4-Hydroxyquinoline-3-carbonyl)-N-p-} tolylhydrazinecarboxamide (7d)

Yield, 40\%; white powder; $\mathrm{mp} 320^{\circ} \mathrm{C}$ (decomposed); IR ( $\mathrm{KBr}$ disk): $v\left(\mathrm{~cm}^{-1}\right)$ 1400-1600 (aromatic), 1620-1670 (C=O), 2900-3500 (O-H), 3220 \& $3280(\mathrm{~N}-\mathrm{H})$; ${ }^{1} \mathrm{HNMR}$ (DMSO-d6, $500 \mathrm{MHz}$ ): $\delta 2.19\left(\mathrm{~s}, 3 \mathrm{H}, \mathrm{CH}_{3}\right), 7.00$ (d, $2 \mathrm{H}, \mathrm{N}-\mathrm{p}$-tolyl $\mathrm{H}_{3} \& \mathrm{H}_{5}$, $J=8.11 \mathrm{~Hz}$ ), 7.29 (d, 2H, p-tolyl $\mathrm{H}_{2} \& \mathrm{H}_{6}, J=8.26 \mathrm{~Hz}$ ), 7.46-7.49 (t, quinoline $\left.\mathrm{H}_{6}, J=7.40 \mathrm{~Hz}\right), 7.69\left(\mathrm{~d}, 1 \mathrm{H}\right.$, quinoline $\left.\mathrm{H}_{8}, J=8.21 \mathrm{~Hz}\right)$, 7.74-7.77 (t, $1 \mathrm{H}$, quinoline $\left.\mathrm{H}_{7}, J=7.27 \mathrm{~Hz}\right), 8.26(\mathrm{~d}, 1 \mathrm{H}$, quinoline $\left.\mathrm{H}_{5}, \mathrm{~J}=8.04 \mathrm{~Hz}\right), 8.33(\mathrm{~s}, 1 \mathrm{H}, \mathrm{N}-\mathrm{H}), 8.72-8.75$ (d, 2H, quinoline $\mathrm{H}_{2}$ \& N-H), 11.36 (s, 1H, N-H), 12.78 (br s, 1H, enolic OH); LC-MS (ESI) $\mathrm{m} / \mathrm{z}$ : $356.82(\mathrm{M}+1,100)$; Anal. Calcd. for $\mathrm{C}_{18} \mathrm{H}_{16} \mathrm{~N}_{4} \mathrm{O}_{3}$ : C, 64.28; H, 4.79; N, 16.66. Found: C, 64.39; H, 4.66; N, 16.77.

\section{2-(4-Hydroxyquinoline-3-carbonyl)-N-(4- \\ methoxyphenyl) hydrazine carboxamide (7e)}

Yield, 35\%; white powder; mp 265-266 ${ }^{\circ} \mathrm{C}$; IR ( $\mathrm{KBr}$ disk): $\mathrm{v}\left(\mathrm{cm}^{-1}\right)$ 1400-1600 (aromatic), 1650-1680 (C=O), 2800-3500 (O-H), 3300, 3360 \& 3460 (N-H); ${ }^{1} \mathrm{HNMR}$ (DMSO-d6, 500MHz): $\delta 3.66$ (s, $\left.3 \mathrm{H}, \mathrm{OCH}_{3}\right), 6.80$ (d, 2H, N-4-methoxyphenyl $\mathrm{H}_{3} \& \mathrm{H}_{5}, J=8.94 \mathrm{~Hz}$ ), 7.31 (d, 2H, N-4-methoxyphenyl $\mathrm{H}_{2} \& \mathrm{H}_{6}, J=8.94 \mathrm{~Hz}$ ), 7.46-7.49 (t, quinoline $\left.\mathrm{H}_{6}, J=7.84 \mathrm{~Hz}\right), 7.69\left(\mathrm{~d}, 1 \mathrm{H}\right.$, quinoline $\mathrm{H}_{8}, J=8.20 \mathrm{~Hz}$ ), 7.74-7.77 (t, $1 \mathrm{H}$, quinoline $\left.\mathrm{H}_{7}, \mathrm{~J}=8.26 \mathrm{~Hz}\right), 8.26\left(\mathrm{~d}, 1 \mathrm{H}\right.$, quinoline $\mathrm{H}_{5}$, $J=8.09 \mathrm{~Hz}), 8.29(\mathrm{~s}, 1 \mathrm{H}, \mathrm{N}-\mathrm{H}), 8.65(\mathrm{~s}, 1 \mathrm{H}, \mathrm{N}-\mathrm{H}), 8.73(\mathrm{~s}, 1 \mathrm{H}$, quinoline $\mathrm{N}-\mathrm{H}$ ), 11.34 (s, 1H, N-H), 12.78 (br s, 1H, enolic OH); LC-MS (ESI) m/z: $352.83(\mathrm{M}+1,100)$; Anal. Calcd. for $\mathrm{C}_{18} \mathrm{H}_{16} \mathrm{~N}_{4} \mathrm{O}_{4}$ : C, 61.36; $\mathrm{H}$, 4.58; N, 15.90. Found: C, 61.44; H, 4.46; N, 15.87 .

\section{In-vitro anti-HIV and cytotoxicity assays}

The inhibitory effect of compounds against HIV-1 was studied by single cycle replication assay as previously described [20]. In brief, Hela cells $\left(6 \times 10^{3}\right.$ per well of 96 -wells plate) were infected with single cycle replicable HIV NL4-3 virions (200 ng $\mathrm{P}_{24}$ ) in the presence of different concentrations of compounds (1, 10 and $100 \mu \mathrm{M})$. Addition of compounds to the cells environment was simultaneous with viral infection. The supernatants were collected $72 \mathrm{~h}$ postinfection and evaluated for $\mathrm{P}_{24}$ antigen load by capture ELISA (Biomerieux, France). The inhibition rate (\%) of $\mathrm{P}_{24}$ expression was calculated. The cellular toxicity was evaluated by XTT (sodium 3-[1(phenylaminocarbonyl)-3,4-tetrazolium]bis(4-methoxy-6-nitro)benzene sulfonic acid) proliferation assay (Roche, Germany) according to the kit instruction [21,22]. The HIV replication assay plates were directly considered for cytotoxicity assay after determination of $\mathrm{P}_{24}$ load.

\section{Molecular modeling (docking) studies}

The active compounds were selected for docking studies against HIV-1 IN. 3OYA is used for binding mode analysis of HIV-1 IN inhibitory activity. All the compounds were built using ChemDraw and subsequently minimized. The protein structure was prepared for docking using AUTODOCK Tool. Docking was performed by AutoDock 4.0 program, using the implemented empirical free energy function and the Lamarckian Genetic
Algorithm (LGA) [23]. Co-crystallized ligand and all water molecules were removed from crystal protein (3OYA) while magnesium ions $\left(\mathrm{Mg}^{2+}\right)$ at the active site of HIV-1 IN were maintained. Polar hydrogens were added and non polar hydrogens were merged, finally Kallman united atom charge and atom type parameter was added to 3OYA. Grid map dimensions $(20 \times 20 \times 20)$ were set surrounding active site. Lamarckian genetic search algorithm was employed and docking run was set to 50 .

\section{Results and Discussion}

$\nabla$

\section{Chemistry}

The target 4-hydroxyquinoline-3-carbohydrazide derivatives were synthesized via the route outlined in 0 Fig. 2 . The GouldJacob cyclization reaction is regarded as the most fundamental and versatile method to form the core structure of 4-hydroxyquinoline-3-carboxylate [19]. The compounds 4-hydroxy-N'-(4substituted-benzoyl) quinoline-3-carbohydrazides 6a-e and $\mathrm{N}$-(4-substituted-phenyl)-2-(4-hydroxyquinoline-3-carbonyl) hydrazinecarboxamide 7a-e were prepared according to GouldJacob cyclization reaction, starting from aniline in 4 steps. Condensation of aniline with ethoxymethylene malonate diethyl ester (EMME) yielded diethyl 2-((phenylamino)-methylene) malonate 3. Compound 3 was converted to ethyl 4-hydroxyquinoline-3-carboxylate 4 in $\mathrm{Ph}_{2} \mathrm{O}$ containing catalytic 2-chlorobenzoic acid. Compound $\mathbf{4}$ was subsequently treated with hydrazine in DMF to form the corresponding hydrazide intermediate $\mathbf{5}$. Compound $\mathbf{5}$ was used as a key intermediate for obtaining 2 different series of compounds. In the first series, to synthesize 4-hydroxy-N'-(4-substituted-benzoyl)quinoline-3-carbohydrazides 6a-e, compound $\mathbf{5}$ reacted with 4-substituted benzoyl chlorides in presence of catalytic amount of $\mathrm{Na}_{2} \mathrm{CO}_{3}$ in DMF. In the second series, compounds $7 \mathbf{a}-\mathbf{e}$ were synthesized by reaction of hydrazide intermediate $\mathbf{5}$ with 4 -substituted phenyl isocyanate in $\mathrm{MeCN}$. All compounds were stable and pure and their chemical structures were confirmed by IR, ${ }^{1} \mathrm{HNMR}$ and ESI-MS.

\section{Biological evaluation}

The anti-HIV activity of the all compounds was measured by determining their ability to general inhibition of the replication of HIV-1 in Hela cells cultures. For comparative purposes, nucleoside reverse transcriptase inhibitor, AZT, was assayed in the same cells. All synthesized compounds were also tested for their cytotoxicity by MTT assay. As shown in 0 Table 1, all synthesized compounds displayed no significant cytotoxicity at concentration of $100 \mu \mathrm{M}$. Most of the tested compounds produced inhibitory effects at $100 \mu \mathrm{M}$ concentration with a percentage ranging from 5 to $32 \%$. However, in all cases, the measured activities were lower than that of AZT. These results indicated that chelation of the $2 \mathrm{Mg}^{2+}$ cations by adjacent carboxylic and hydroxyl groups as chelation motif are not strong enough to allow for a strong anti-HIV-1 activity by these compounds. 4-Hydroxyquinoline-3-carbohydrazide compounds 6a-e containing 4-substituted benzoyl groups as aromatic ring, showed moderate activity against HIV ranging from $21-32 \%$, except for compound $\mathbf{6 c}$, which exhibited $8 \%$ inhibition rate. However, compounds 7a-e having 4-substituted N-phenyl moiety displayed no considerable anti-HIV activity (0-5\%) except for compound 7e (having methoxy substitution) with inhibition rate of $28 \%$. The structural difference between $\mathbf{6 a - e}$ and $\mathbf{7 a - e}$ lies in the incorporation of $\mathrm{N}-\mathrm{H}$ between substituted phenyl ring 


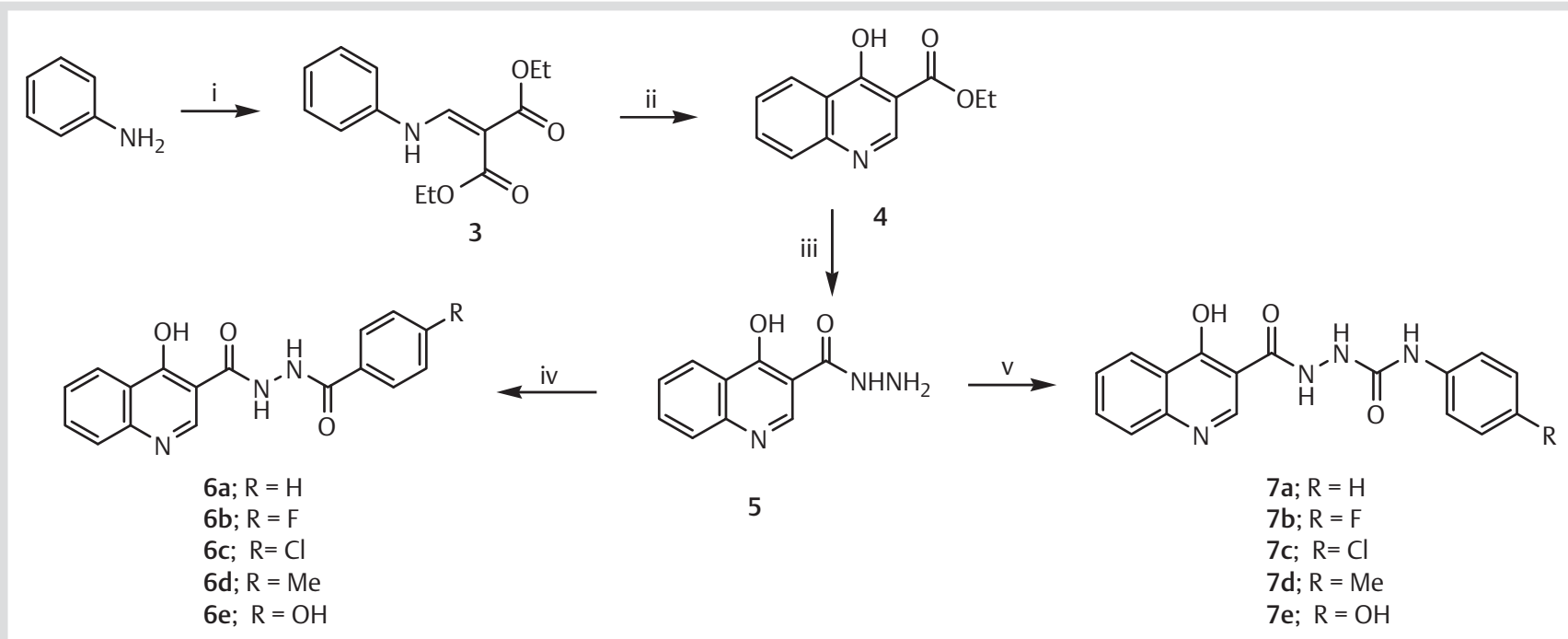

Fig. 2 Reagents and conditions: (i) EMME, $120^{\circ} \mathrm{C}, 1 \mathrm{~h}$ (ii) 2-chlorobenzoic acid, $\mathrm{Ph}_{2} \mathrm{O}, 250^{\circ} \mathrm{C}, 2 \mathrm{~h}$ (iii) $\mathrm{NH}_{2} \mathrm{NH}_{2} \cdot \mathrm{OH}, \mathrm{DMF}$, rt, $12 \mathrm{~h}$ (iv) 4-substituted benzoyl chloride, $\mathrm{Na}_{2} \mathrm{CO}_{3}$, DMF, rt, $16 \mathrm{~h}$ (v) 4-substituted phenyl isocyanate, MeCN, rt, $16 \mathrm{~h}$.

and second carbonyl group in compounds 7a-e. These results demonstrated that specific orientation of phenyl ring to the chelator group is a requirement for high anti-HIV-1 activity. According to our results compound $6 \mathrm{~d}$ having methyl group at para position of phenyl ring showed the highest anti-HIV-1 activity (32\%) among the synthesized compounds. These findings conform to the common pharmacophore of HIV-1 integrase inhibitors in which a hydrophobic aromatic group with a specific spatial arrangement to a chelator is a required structural determinant.

\section{Molecular modeling studies}

Docking studies were performed to predict the binding mode of designed compounds and to rationalize the observed structure activity relationships. Based on these results, a mechanism of inhibition for these compounds is proposed. Due to the lack of HIV-1 IN/DNA experimental structures, the building of a reliable model is essential for predicting docking of integrase inhibitors. Hare et al. solved the crystal structures of full-length prototype foamy virus (PFV) integrase DNA complexes with various HIV-1 integrase inhibitors, exhibiting 2-metal ions in the strand transfer active site $\left(\mathrm{Mg}^{2+}\right.$ or $\left.\mathrm{Mn}^{2+}\right)$ [24]. As shown by Hare et al. PFV integrase can be considered as a good model for the development of HIV-1 integrase strand transfer inhibitors. The secondary structures of PFV IN (PDB: 3OYA) and HIV-1 IN catalytic core domain (PDB: 1BL3) have highly conserved architectures, with a calculated RMSD of $1.04 \AA$. $2 \mathrm{Mg}^{2+}$ ions in PFV integrase are coordinated by Asp128, Asp185 and Glu221 which are corresponded to HIV-1 integrase Asp64, Asp116 and Glu152, respectively. On the basis of these considerations, we decided to undertake docking studies of our molecules based on the 30YA X-ray crystallographic structure of PFV IN. Docking of the most potent compounds (6d and 7e) in the newly built model was performed with the AUTODOCK 4.2 program. All these compounds showed a similar interfacial-binding mode in which the adjacent carboxylic and hydroxyl group at position of C-3 and $\mathrm{C}-4$ of quinoline ring moiety chelate the $\mathrm{Mg}^{2+}$ ions (see docking result of $\mathbf{6 d}$ and 7e, $\odot$ Fig. 3). The displaced 3'-adenosine terminal base (A17) was involved in a $\pi$-stacking interaction with the quinoline ring. The $p$-substituted-phenyl group fitted within a tight pocket formed by cytosine 16 (C16), guanine 4 (G4). Fur-
Table 1 Anti-HIV activity of synthesized compounds.<smiles>NNC(=O)Nc1cnc2ccccc2c1O</smiles>

\begin{tabular}{|lllll} 
Compound & $\mathbf{X}$ & $\mathbf{R}$ & \multicolumn{2}{c}{$\mathbf{1 0 0} \boldsymbol{\mu M}$} \\
& & & $\begin{array}{l}\text { (\%) inhibition rate of } \\
\mathbf{P}_{\mathbf{2 4}} \text { expression }\end{array}$ & $\begin{array}{l}\text { \% Cell } \\
\text { viability }\end{array}$ \\
\hline 6a & - & $\mathrm{H}$ & 21.1 & 97.3 \\
\hline 6b & - & $\mathrm{F}$ & 22.7 & 79.1 \\
\hline 6c & - & $\mathrm{Cl}$ & 7.9 & 70.0 \\
$\mathbf{6 d}$ & - & $\mathrm{CH}_{3}$ & 31.7 & 81.1 \\
\hline 6e & - & $\mathrm{OCH}_{3}$ & 22.9 & 76.9 \\
\hline 7a & $\mathrm{NH}$ & $\mathrm{H}$ & - & 81.3 \\
\hline 7b & $\mathrm{NH}$ & $\mathrm{F}$ & 5.8 & 73.1 \\
\hline 7c & $\mathrm{NH}$ & $\mathrm{Cl}$ & - & 86.7 \\
\hline 7d & $\mathrm{NH}$ & $\mathrm{CH}_{3}$ & $\mathrm{ND}$ & 84.0 \\
\hline 7e & $\mathrm{NH}$ & $\mathrm{OCH}_{3}$ & 28.0 & 98.0 \\
\hline AZT & & & 100 & 100 \\
\hline Ralvitegravir & & & 100 & 100 \\
\hline
\end{tabular}

*ND: Not determined

ther docking study revealed that active compounds ( $6 \mathbf{d}$ and $7 \mathbf{7 e})$ occupied same space near to $\mathrm{Mg}^{2+}$ ions as Raltegravir ( $\bullet$ Fig. 4). These docking results were in agreement with the common structure activity relationships of HIV-1 integrase inhibitors.

\section{Conclusions}

$\nabla$

In conclusion, we have designed and synthesized a novel class of 4-hydroxyquinoline-3-carbohydrazide derivatives by merging 2 well-known anti-HIV-1 scaffolds, salicylhydrazide and 4-quinolone-3-carboxylic acid. The major significance of this work as compared to previous reports on salicylhydrazide structure is that the synthesized compounds displayed no considerable cytotoxicity in cells cultures. So, this novel scaffold is suitable for the 


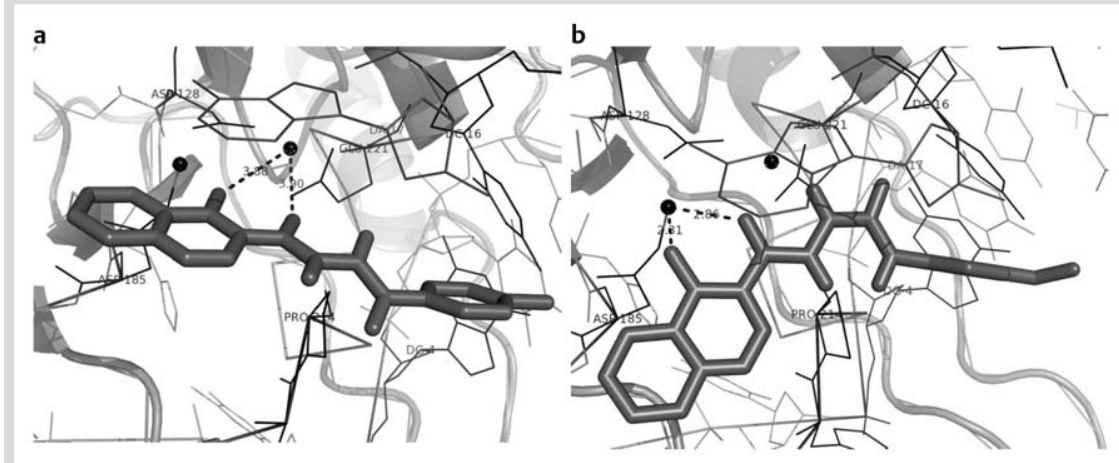

Fig. 3 Mode of binding of $\mathbf{6 d}(\mathbf{a})$ and $7 \mathbf{7 e}(\mathbf{b})$ in the active site of HIV-1 IN.
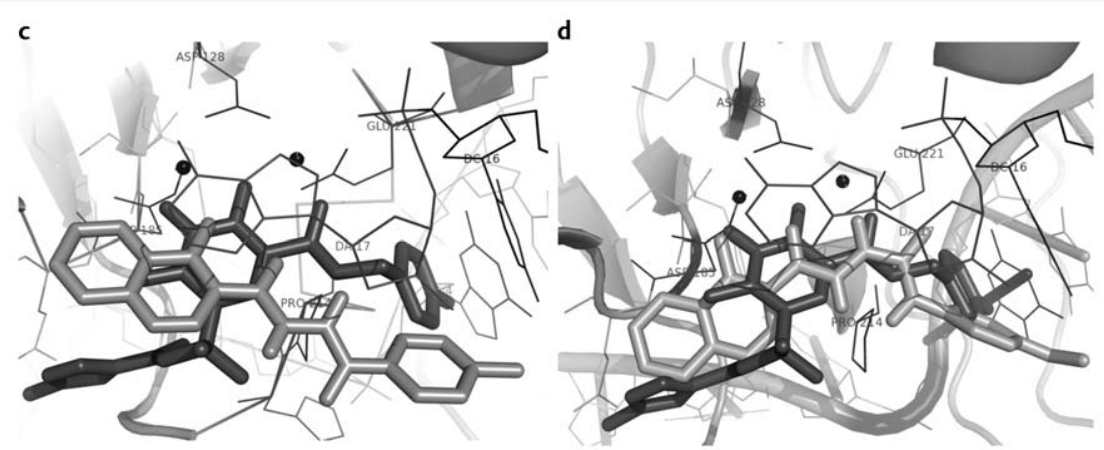

Fig. 4 Compound $\mathbf{6 d}$ and $7 \mathrm{e}$ superimposed on the Raltegravir molecule (c and $\mathbf{d}$ respectively).

development of new hits. Compounds $\mathbf{6} \mathbf{d}$ and 7e were found to be the most active in cell based anti-HIV assay with inhibition rates of 32 and $28 \%$ at concentration of $100 \mu \mathrm{M}$, respectively. The molecular modeling studies suggested that the anti-HIV activity of these compounds might involve a metal chelating mechanism.

\section{Acknowledgement \\ $\nabla$}

We like to thank Deputy of Research, School of Pharmacy, Shahid Beheshti University of Medical Sciences for financial support of this work as part of Ph.D thesis of Zahra Hajimahdi.

\section{Conflict of Interest}

$\nabla$

The authors have declared no conflict of interest.

\section{References}

1 Palella A, Delaney FJ, Moorman KM et al. Declining morbidity and mortality among patients with advanced human immunodeficiency virus infection. HIV outpatient study investigators. New Engl J Med 1998; 338: 853-860

2 Richman DD. HIV chemotherapy. Nature 2001; 410: 995-1001

3 Esposit $D$, Craigie R. HIV integrase structure and function. Adv Virus Res 1999; 52: 319-333

4 Chen IJ, Neamati N, MacKerell AD. Structure-based inhibitor design targeting HIV-1 integrase. Curr Drug Targets Infect Disord 2002; 2: 217-234

5 Miller MD, Farnet CM, Bushman FD. Human immunodeficiency virus type 1 preintegration complexes: studies of organization and composition. J Virol 1997; 71: 5382-5390

6 Colicelli J, Goff SP. Sequence and spacing requirements of a retrovirus integration site. J Mol Biol 1988; 199: 47-59

7 Pommier Y, Johnson AA, Marchand C. Integrase inhibitors to treat HIV/ AIDS. Nat Rev Drug Discovery 2005; 4: 236-248

8 Johns BA, Svolto AC. Advances in two-metal chelation inhibitors of HIV integrase. Expert Opin Ther Pat 2008; 18: 1225-1237
9 Dayam R, Al-Mawsawi LQ Neamati N. HIV-1 integrase inhibitors: An emerging clinical reality. Drugs R D 2007; 8: 155-168

10 Shimura K, Kodama E, Sakagami $Y$ et al. Broad antiretroviral activity and resistance profile of the novel human immunodeficiency virus integrase inhibitor elvitegravir (JTK-303/GS-9137). J Virol 2008; 82: 764-774

11 Min S, Song I, Borland J et al. Pharmacokinetics and safety of S/ GSK1349572, a nextgeneration HIV integrase inhibitor, in healthy volunteers. Antimicrob Agents Chemother 2010; 54: 254-258

12 Charpentier C, Karmochkine M, Laureillard D et al. Drug resistance profiles for the HIV integrase gene in patients failing raltegravir salvage therapy. HIV Med 2008; 9: 765-770

13 Zhao H, Neamati N, Sunder S et al. Hydrazide-containing inhibitors of HIV-1 integrase. J Med Chem 1997; 40: 937-941

14 Neamati $N$, Hong $H$, Owen JM et al. Salicylhydrazine-containing inhibitors of HIV-1 integrase: implication for a selective chelation in the integrase active site. J Med Chem 1998; 41: 3202-3209

15 Pommer Y, Marchand C, Neamati N. Retroviral integrase inhibitors year 2000: update and perspectives. Antiviral Res 2000; 47: 139-148

16 Al-Mawsawi LQ Dayam R, Taheri $L$ et al. Discovery of novel noncytotoxic salicylhydrazide containing HIV-1 integrase inhibitors. Bioorg Med Chem Lett 2007; 17: 6472-6475

17 Cecchetti V, Parolin C, Moro $S$ et al. 6-Amino quinolones as new potential anti-HIV agents. J Med Chem 2000; 43: 3799-3802

18 Tabarrini O, Massari S, Daelemans D et al. Structure-activity relationship study on anti-HIV 6-desfluoroquinolones. J Med Chem 2008; 51: $5454-5458$

19 Gould RG, Jacobs WA. The synthesis of certain substituted quinolines and 5, 6-benzoquinolines. J Am Chem Soc 1939; 61: 2890-2895

20 Zabihollahi $R$, Sadat SM, Vahabpour $R$ et al. Development of singlecycle replicable human immunodeficiency virus 1 mutants. Acta Virol 2011; 55: 15-22

21 Scudiero DA, Shoemaker RH, Paull KD et al. Evaluation of a soluble tetrazolium/formazan assay for cell growth and drug sensitivity in culture using human and other tumor cell lines. Cancer Res 1998; 48: 4827-4833

22 Lin CC, Cheng HY, Yang CM et al. Antioxidant and antiviral activities of the Euphorbia thymifolia Linn. J Biomed Sci 2002; 9: 656-664

23 Morris GM, Goodsell DS, Halliday RS et al. Automated docking using a Lamarckian genetic algorithm and an empirical binding free energy function. J Comput Chem 1998; 19: 1639-1662

24 Hare S, Gupta SS, Valkov E et al. Retroviral intasome assembly and inhibition of DNA strand transfer. Nature 2010; 464: 232-236 